\title{
A citação da Revista Portuguesa de Clínica Geral na Revista Portuguesa de Clínica Geral
}

Inês Rosendo,* Tiago Santos,* Dina Martins, * Gonçalo Pimenta,* Maria da Glória Neto, ** Maria dos Prazeres Francisco, ${ }^{* *}$ Luiz Miguel Santiago***

\section{RESUMO}

Objectivos: A Revista Portuguesa de Clínica Geral (Revista) é a publicação mais prestigiada na área, em Portugal. Assim sendo é importante conhecer a citação bibliográfica de artigos já publicados noutros números desta Revista. Pelo que o objectivo do presente estudo é medir a citação de artigos já publicados na Revista Portuguesa de Clínica Geral.

Tipo de estudo: Estudo observacional, transversal e descritivo.

População e Métodos: Revisão sistemática de todos os números da Revista quanto ao tipo de artigo, número total de referências bibliográficas, número de referências bibliográficas de artigos publicados em Portugal e número de referências bibliográficas de artigos já publicados na Revista, entre Janeiro de 2000 e Dezembro de 2006, inclusive.

Resultados: Entre 2000 e 2006, em 357 artigos, as referências totais nos artigos publicados foram de 16,4ะ15,5, as de artigos

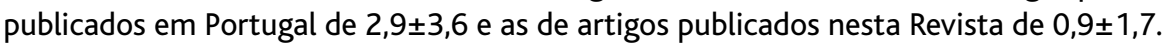

Indicadores: Número de citações de artigos publicados na Revista / Número total de citações bibliográficas na Revista = 0,05; Número de citações de artigos publicados na Revista / Número total de citações bibliográficas portuguesas na Revista = 0,30; e Número de citações de artigos publicados na Revista / Número de artigos publicados na Revista, de 1,7 e 0,27 para os anos de 2001 e 2003.

Conclusões: Sendo baixa a citação de artigos publicados na Revista Portuguesa de Clínica Geral, parece ser necessário que os médicos de Clínica Geral a leiam para suporte à realização de trabalhos e sua discussão. Propõe-se a monitorização futura desta realidade. Propõe-se ainda a indexação que poderá ser uma forma de obviar este problema.

Palavras-chave: Cuidados de Saúde Primários, Clínica Geral, Referências Bibliográficas, Artigos.

\section{INTRODUÇÃO}

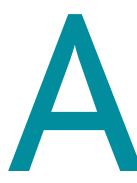
qualidade de uma revista médica pode ser objectivada por vários indicadores de citação ou referenciação bibliográfica, de entre os quais o Índice Imediato, que reflecte a proporção de artigos citados/publicados numa publicação num determinado ano. ${ }^{1}$ Deve, no entanto, haver rigoroso cuidado para que sejam observados critérios constantes e de elevado nível de apreciação nos artigos submetidos a publicação, sendo seguidas regras consensuais e publicadas. ${ }^{2}$ Do acima referido podem-se depreender critérios de frequência e de qualidade de-

\footnotetext{
*Médico, Interno de Medicina Geral e Familiar, Centro de Saúde de Eiras.

**Assistente Graduada de Clínica Geral, Centro de Saúde de Eiras

***Mestre em Medicina, Chefe de Serviço de Clínica Geral,

Centro de Saúde de Eiras
}

vendo, mesmo assim, haver o cuidado de relativizar os indicadores em função do tipo de artigos publicados e que podem ser inovações, meras revisões ou até só «mais do mesmo». ${ }^{3}$

Em Portugal, a Revista Portuguesa de Clínica Geral (Revista) é a mais prestigiada e conhecida revista que versa os vários capítulos da produção científica portuguesa da especialidade de Medicina Geral e Familiar. Assim sendo, deve ser medida a sua importância, pelo menos como repositório da referenciação de artigos portugueses feitos por médicos de Clínica Geral e por eles citados aquando de novas publicações na Revista. Pelo que o objectivo do presente estudo é medir a citação de artigos publicados na Revista Portuguesa de Clínica Geral.

Mais especificamente medir o peso da citação de ar- 
tigos publicados previamente na Revista, nos artigos publicados entre os anos 2000 a 2006.

\section{MÉTODOS}

Universo: Todos os artigos de todos os números da Revista de 2000 a 2006, inclusive. Médicos revisores dos artigos. Ficheiros de dados em Excel e SPSS, versão 11.0. Metodologia: Trabalho observacional, transversal e descritivo, pela revisão sistemática de todos os números da Revista quanto ao tipo de artigo, número total de referências bibliográficas, número de referências bibliográficas de artigos publicados em Portugal e número de referências bibliográficas de artigos já publicados na Revista.

Indicadores de citação ou referenciação bibliográfica a serem aplicados no geral e por secção da Revista:

1. Número de citações de artigos publicados na Revista/Número total de citações bibliográficas na Revista.

2. Número de citações de artigos publicados na Revista/Número total de citações bibliográficas portuguesas na Revista.

3. Por ano: Número de citações de artigos publicados na Revista/Número de artigos publicados na Revista. Foram consideradas para esta análise as categorias definidas pela revista. ${ }^{4}$

\section{RESULTADOS}

Num total de 357 artigos, segundo o Quadro I, verificamos que os artigos na secção Dossier representam $27 \%$ do total analisado, logo seguidos dos artigos originais, com uma frequência relativa de $24 \%$.

Para os 357 artigos revistos, encontramos os valores de referenciação bibliográfica constantes do Quadro II, devendo ser referida uma proporção de 0,05 para referências da Revista/referências totais e de 0,17 para referências portuguesas/referências totais.

No Quadro III mostramos a distribuição média de referências bibliográficas por tipo de artigo ao longo do período em estudo. Para as categorias mais representativas é de salientar a menor frequência de referenciação nos artigos originais, nas publicações de casos e nos artigos da secção Dossier.

O resultado dos indicadores desenhados para esta análise é apresentado no Quadro IV, sendo de assinalar dois picos de maior proporção nos anos de $2001 \mathrm{e}$ 2005, para o Indicador 3.

A dinâmica de crescimento ao longo dos anos e entre o início e o final do período em analise é mostrada no Quadro V, sendo de realçar um crescimento negativo entre 2000 e 2006.

No Quadro VI são mostrados os valores obtidos para o Indicador 3, por tipo de artigo e por ano. De realçar que, para os anos 2000 a 2002, não havia a secção Dossier e que as secções de Revisão, Formação, Prática e Relatos de Casos têm um reduzido número de artigos por ano.

\section{DISCUSSÃO}

A afirmação de uma especialidade médica depende da massa crítica de conhecimento que ela consegue desenvolver e objectivamente publicar. Não encontrámos resultados quanto a esta temática em Portugal e, em particular, quanto à área em estudo. Deliberadamente decidimos realizar um trabalho de avaliação quantitativa e por área de publicação da Revista.

Apesar de podermos pensar que o tempo de exposição seja ainda curto e não tendo sido utilizados artigos anteriores a 2000 por dificuldades logísticas várias, os resultados agora obtidos mos- 
QUADRO II. Referências totais, portuguesas e de artigos previamente publicados na Revista no período de 2000-2006

\begin{tabular}{l|c|c|c} 
& $\begin{array}{c}\text { Referências } \\
\text { totais }\end{array}$ & $\begin{array}{c}\text { Referências } \\
\text { portuguesas }\end{array}$ & $\begin{array}{c}\text { Referências } \\
\text { da Revista }\end{array}$ \\
\hline Média $\pm \mathrm{dp}$ & $16,42 \pm 15,5$ & $2,88 \pm 3,64$ & $0,87 \pm 1,73$ \\
\hline Mediana & 12 & 2 & 0 \\
\hline Moda & 7 & 0 & 0 \\
\hline
\end{tabular}

QUADRO III. Média de referências bibliográficas por tipo de artigo ao longo do período considerado

\begin{tabular}{l|c|c|c} 
Tipo de artigo & $\begin{array}{c}\text { Referências } \\
\text { totais }\end{array}$ & $\begin{array}{c}\text { Referências } \\
\text { portuguesas }\end{array}$ & $\begin{array}{c}\text { Referências } \\
\text { da Revista }\end{array}$ \\
\hline Editorial $(n=78)$ & $7,6 \pm 5,4$ & $3,0 \pm 2,9$ & $1,9 \pm 2,3$ \\
\hline Original $(n=87)$ & $16,26 \pm 9,2$ & $3,8 \pm 3,1$ & $0,9 \pm 1,6$ \\
\hline Revisão $(n=40)$ & $18,5 \pm 25,5$ & $21,3 \pm 2,5$ & $0,23 \pm 0,6$ \\
\hline Formação $(n=6)$ & $17,3 \pm 15,0$ & $2,7 \pm 1,5$ & $0,5 \pm 0,84$ \\
\hline Prática $(n=7)$ & $30,9 \pm 26,5$ & $9,4 \pm 8,9$ & $2,1 \pm 4,4$ \\
\hline $\begin{array}{l}\text { Relato de casos } \\
\text { (n=9) }\end{array}$ & $9,7 \pm 4,9$ & $0,6 \pm 0,9$ & $0,1 \pm 0,3$ \\
Dossier $(n=95)$ & $18,7 \pm 16,3$ & $1,9 \pm 3,9$ & $0,1 \pm 0,4$ \\
\hline Opinião $(n=35)$ & $12,4 \pm 10,4$ & $2,8 \pm 3,9$ & $2,9 \pm 1,5$
\end{tabular}

tram uma muito baixa citação de artigos anteriormente publicados na Revista, havendo uma flutuação ao longo do tempo que pode reflectir, eventualmente, a entrada de novas equipas redactoriais. No entanto e porque não dispomos de dados quanto a períodos anteriores de publicação da Revista, seremos muito prudentes quanto a esta discussão.

A explicação destes resultados levanta várias hipóteses de raciocínio:

- A Revista não publica artigos que reflictam assuntos sobre os quais os Médicos de Clínica Geral produzem ou a temática dos novos artigos nada tem a ver com os já publicados. Tal não parece ser o caso, pois os artigos publicados têm relação com alguns anteriormente publicados quanto a fundamentação, a explicação de resultados e sua discussão, bem como a sugestões para a prática.

- A Revista é pouco lida e acedida e, como tal, os seus artigos não são conhecidos. Não podemos discutir

\begin{tabular}{|c|c|c|c|}
\hline Ano/Indicador & Indicador 1 & Indicador 2 & Indicador 3 \\
\hline 2000 & 0,06 & 0,35 & 1,00 \\
\hline 2001 & 0,11 & 0,53 & 1,70 \\
\hline 2002 & 0,06 & 0,25 & 0,86 \\
\hline 2003 & 0,02 & 0,29 & 0,27 \\
\hline 2004 & 0,04 & 0,19 & 0,52 \\
\hline 2005 & 0,07 & 0,31 & 1,18 \\
\hline 2006 & 0,03 & 0,24 & 0,84 \\
\hline Total & 0,05 & 0,30 & 0,87 \\
\hline
\end{tabular}

Nota: 1. Número de citações de artigos publicados na Revista / Número total de citações bibliográficas na Revista;

2. Número de citações de artigos publicados na Revista / Número total de citações bibliográficas portuguesas na Revista;

3. Número de citações de artigos publicados na Revista / Número de artigos publicados na Revista.

QUADRO V. Dinâmica de crescimento $(\Delta)$ dos vários indicadores em estudo ao longo do período considerado

\begin{tabular}{l|c|c|c}
$\Delta$ /Indicador & Indicador $\mathbf{1}$ & Indicador $\mathbf{2}$ & Indicador 3 \\
\hline $2000 / 2001$ & 0,69 & 0,51 & 0,70 \\
\hline $2002 / 2003$ & $-0,48$ & $-0,53$ & $-0,49$ \\
\hline $2003 / 2004$ & $-0,60$ & 0,15 & $-0,69$ \\
\hline $2003 / 2004$ & 0,67 & $-0,33$ & 0,93 \\
\hline $2004 / 2005$ & 0,92 & 0,64 & 1,25 \\
\hline $2005 / 2006$ & $-0,52$ & $-0,23$ & $-0,28$ \\
\hline $2000-2006$ & $-0,46$ & $-0,32$ & $-0,16$ \\
\hline
\end{tabular}

Nota: 1. Número de citações de artigos publicados na Revista / Número total de citações bibliográficas na Revista;

2. Número de citações de artigos publicados na Revista / Número total de citações bibliográficas portuguesas na Revista;

3. Número de citações de artigos publicados na Revista / Número de artigos publicados na Revista.

esta hipótese por inexistência de dados ou resultados. No entanto, a Revista é distribuída gratuitamente a todos os sócios da Associação Portuguesa dos Médicos de Clínica Geral.

- A Revista é de difícil pesquisa e não tem os artigos publicados em motores de busca. O Índex Medicus, ao permitir acesso a títulos, autores e número de Revista, contraria, em parte, este argumento. Outros motores de busca como «Yahoo» e «Google» também permitem o acesso a artigos da Revista. 


\begin{tabular}{|c|c|c|c|c|c|c|c|c|}
\hline Ano/Tipo & Editorial & Original & Revisão & Formação & Prática & Relato & Dossier & Opinião \\
\hline 2000 & 0,29 & 0,34 & 0,14 & 0,06 & 0,00 & 0,03 & 0,00 & 0,14 \\
\hline 2001 & 0,18 & 0,16 & 0,12 & 0,03 & 0,03 & 0,00 & 0,00 & 0,07 \\
\hline 2002 & 0,22 & 0,47 & 0,19 & 0,00 & 0,03 & 0,03 & 0,00 & 0,22 \\
\hline 2003 & 0,75 & 0,63 & 0,38 & 0,00 & 0,00 & 0,06 & 1,50 & 0,38 \\
\hline 2004 & 0,75 & 0,75 & 0,44 & 0,06 & 0,00 & 0,13 & 1,56 & 0,25 \\
\hline 2005 & 0,15 & 0,20 & 0,08 & 0,02 & 0,03 & 0,03 & 0,30 & 0,05 \\
\hline 2006 & 0,26 & 0,24 & 0,04 & 0,00 & 0,04 & 0,04 & 0,48 & 0,09 \\
\hline
\end{tabular}

- A exigência científica dos novos autores de artigos em Clínica Geral Portuguesa é superior à da produção portuguesa na Revista. Seria um bom argumento explicativo não fosse dar-se o caso de que um percurso histórico evocativo deve fazer parte da ciência e que, no passado, foi produzida ciência, com a visão e os métodos que determinaram que o corpo revisor e editorial da Revista a considerasse publicável.

- A Revista, não estando indexada, não tem a representatividade pretendida pelos autores de artigos e, assim, é pouco citada. Este é um senão, um argumento de peso, para quem apenas pesquise em motores ou em livrarias «on-line» nas quais, de facto, a Revista não tem estado.

- A Revista não tem credibilidade perante a comunidade de Medicina Geral e Familiar Portuguesa. Esta explicação não parece dever ser evocada dado o percurso profissional de todos os que, na época deste estudo e mesmo antes, ocuparam lugares de responsabilidade editorial e de revisão e que são, por si sós, a garantia de honestidade e conhecimento. E tal argumento colide com o facto de médicos de outras especialidades solicitarem publicação na Revista.

Uma vez que a Revista publica artigos em muitas áreas da Clínica Geral/Medicina Familiar, em Portugal designada por Medicina Geral e Familiar, ${ }^{5}$ e é distribuída gratuitamente a todos os médicos inscritos na Associação Portuguesa dos Médicos de Clínica Geral, seria de esperar mais elevados valores dos indicadores ora construídos.

A pesquisa em tempo real, ou «on-line», é morosa e dispendiosa, devendo ser revistos os procedimentos para, no seu sítio na Internet, poderem ser conhecidos quer por matéria, quer por autor, os textos publicados.

Houve tempos em que, após interregno de publicação, foi grande o esforço para repor em marcha este importante instrumento de afirmação da Especialidade com qualidade e com saber fazer implicando a necessidade de maior formação pré e pós-graduada. ${ }^{6}$

É elevado o padrão de qualidade de revisão dos artigos propostos para publicação, ${ }^{5} \mathrm{com}$ grau crescente de exigência, o que torna os artigos publicados em material de elevado valor científico, pelo que se estranha este aparentemente baixo valor da referenciação de artigos publicados na Revista. Encontrámos situações em que artigos não tinham em atenção outros já publicados e que deram direito a carta ao Editor. ${ }^{7}$ Já não estranhamos que tal aconteça na secção Dossier em que, convidados a escrever sobre temas específicos, vindos muitos de fora da área da Clínica Geral, tenham menor citação de artigos da Revista.

O ensino da Clínica Geral/Medicina Familiar em Portugal, quer pré quer pós-graduado, deve também ser suportado em publicações portuguesas, como reflexo da experiência de campo tanto médica como não médica mas aqui afim da Clínica Geral. ${ }^{8,9} \mathrm{Com}$ secções várias e revisões temáticas, de grande qualidade científica, em «Dossier» para estudo bibliográfico, estranham-se os presentes valores.

É que, de facto, a Revista Portuguesa de Clínica Geral tem já um longo repertório de textos importantes na história da Clínica Geral portuguesa, quer conceptuais quer reflexivos, que deveriam ser citados em abundância num exercício de reflexão sobre o contexto português, em vez de apenas serem citados os trabalhos realizados no estrangeiro. ${ }^{10}$ 
Ao longo do período em estudo podemos verificar uma permanente baixa citação de artigos publicados na Revista, mesmo em secções em que a preparação dos escritos implicaria a leitura para a Introdução e para a Discussão, como são os artigos Originais. Também aqui, se for difícil aceder à Revista, a sua citação poderá ser prejudicada, pois haverá sempre tentação de fuga para as citações da Medline, por a pesquisa poder ser fatigante e desmotivadora. ${ }^{11}$ A complexidade das temáticas em Clínica Geral é evidente e a necessidade de sobre elas publicar também. ${ }^{12,13}$ Por estas razões a eventual indexação da Revista poderá ser um caminho que, em definitivo, a imponha.

Podemos então concluir que:

Encontrámos um valor de 0,05, 0,30 e 0,87 respectivamente quanto às razões das referências bibliográficas a artigos publicados na Revista sobre o total de referências, sobre o total de referências nacionais e sobre o número de artigos publicados entre 2000 e 2006.

Parece ser necessário que os médicos de Clínica Geral invistam na leitura da Revista para suporte à realização de trabalhos e sua discussão, bem como uma mais expedita forma de acesso ao publicado na Revista. Será, pois, necessária a monitorização futura desta realidade.

\section{REFERÊNCIAS BIBLIOGRÁFICAS}

1. Opthof T. Sense and nonsense about the impact factor. Cardiovasc Res 1997 Jan; 33 (1): 1-7.

2. Smith R. Peer review: reform or revolution [editorial]. BMJ 1997 Sep

\section{7; 315 (7111): 759-60.}

3. Roncon-Albuquerque JúniorR, Leite-Moreira AF. Índices para avaliação de publicações científicas: o que são e para que servem. Rev Port Cardiol 2000;19 (5): 611-5.

4. Instruções aos Autores. Rev Port Clin Geral 2007 Jan-Fev; 23 (1): 1.

5. Sousa JC. Dois anos com a Revista Portuguesa de Clínica Geral. Rev Port Clin Geral 2007 Jan- Fev; 23 (1): 9-12.

6. Maria VA. Revista Portuguesa de Clínica Geral: dos leitores e para os leitores. Rev Port Clin Geral 2000 Nov-Dez; 16 (6): 419-20.

7. Santiago LM. Obesidade da criança dos 11 aos 13 anos de idade - realidade ou mito. Rev Port Clin Geral 2004 Set-Out; 20 (5): 632-3.

8. Montenegro M. Acessibilidade das publicações periódicas com maior interesse para a Medicina Geral e Familiar. Rev Port Clin Geral 2006 Jul-Ago; 22 (4): 423-30.

9. Monteiro C, Marques FB, Ribeiro CF. Interacções medicamentosas como causa de iatrogenia evitável. Rev Port Clin Geral 2007 Jan-Fev;23(1): 63-73.

10. Sousa JC. A Raposa e as uvas: um olhar sobre a reforma dos cuidados de saúde Primários. Rev Port Clin Geral 2007 Mar-Abr; 23 (2): 117-22.

11. Fonseca CB. Um olhar crítico. Rev Port Clin Geral 2002 Mar-Abr; 18(2): 133.

12. Sá AB. As pontes [editorial]. Rev Port Clin Geral 2002 Mai-Jun;18(3):143-4 .

13. Maria VAJ. Medicina Geral e Familiar: complexidade e riqueza. Rev Port Clin Geral 2000 Jul-Ago; 16 (4): 271-2.

\section{ENDEREÇO PARA CORRESPONDÊNCIA:}

Luiz Miguel Santiago

Quinta de Voimarães, lote $12-5^{\circ} \mathrm{D}$

3000-377 Coimbra

Tlm:+351966 225773

E-mail: Imsantiago@netcabo.pt

Os autores declaram não ter recebido qualquer subvenção para este trabalho ou ter qualquer interesse em outra publicação.

\section{ABSTRACT}

Objective: The Revista Portuguesa de Clínica Geral (Portuguese Journal of General Practice) is the most known and prestiged Portuguese journal on General Practice field. It is therefore important to know about the citation of articles published in the Journal.

Design: In an observational and descriptive work all the numbers and articles published were reviewed by kind of article, total of bibliographic references, total of Portuguese bibliographic references and total of bibliographic references in the Journal, between January 2000 and December 2006. Three quantitative indexes were created.

Results: In 357 articles we found an index of 0,05 for the total of bibliographic references, of 0,30 for the Portuguese bibliographic references and between 1,7 and 0,27 for the years 2001 and 2003 for an index measuring the number of articles in the Journal/number of articles published per year.

Conclusions: In the light of these findings it seems necessary that Portuguese General Practitioners invest on reading the Journal and that a more easy and friendly way can be arranged for searching articles in the Journal.

Keywords. General Practice; Bibliographic References; Articles; Primary Care. 